5 Stochl J, Khandaker GM, Lewis G, Perez J, Goodyer IM, Zammit S, et al. Mood, anxiety and psychotic phenomena measure a common psychopathological factor. Psychol Med 2015; 45: 1483-93.

Golam M. Khandaker, Clinical Lecturer, Department of Psychiatry, University of Cambridge, UK. Email: gmk24@medschl.cam.ac.uk; Claire R. M. Dibben, Consultan Psychiatrist, Norfold and Suffolk NHS Foundation Trust, Bury St Edmunds, UK; Peter B. Jones, Professor, Department of Psychiatry, University of Cambridge, UK

doi: 10.1192/bjp.207.3.271b

Authors' reply: We thank Khandaker et al for their response to our paper, and are in broad agreement that psychotic symptoms are more common than currently recognised in mood disorders. Indeed our previous work has highlighted this and likewise the importance of mood symptoms in psychosis. ${ }^{1,2}$ In our present study the presence of hallucination but not delusion was most frequent in those with a history of child sexual abuse. ${ }^{3}$ Hallucinations in particular may occur in non-psychotic diagnoses in the presence of childhood or later trauma. ${ }^{3}$ Thus hallucinations may have less specificity for psychosis than one might expect given the weight positive symptoms have in diagnostic terms. ${ }^{4,5}$ Khandaker et al's proposal that diagnostic systems should acknowledge the presence of these experiences in non-psychotic disorders is important. This recognition would allow clinicians to better accept diagnostic uncertainty, allay worries provoked by an exclusive association of hallucinations with psychosis, and enable further investigation as to underlying causality within a symptom-specific approach.

1 Hamshere ML, Green EK, Jones IR, Jones L, Moskvina V, Kirov G, et al. Genetic utility of broadly defined bipolar schizoaffective disorder as a diagnostic concept. Br J Psychiatry 2009; 195: 23-9.

2 Upthegrove R, Ross K, Brunet K, McCollum R, Jones L. Depression in firs episode psychosis: the role of subordination and shame. Psychiatry Res 2014; 217: 177-84.

3 Upthegrove $\mathrm{R}$, Chard $\mathrm{C}$, Jones $\mathrm{L}$, Gordon-Smith $\mathrm{K}$, Forty $\mathrm{L}$, Jones I, et al. Adverse childhood events and psychosis in bipolar affective disorder Br J Psychiatry 2015; 206: 191-7.

4 Larøi F, Sommer IE, Blom JD, Fernyhough C, Hugdahl K, Johns LC, et al. The characteristic features of auditory verbal hallucinations in clinical and nonclinical groups: state-of-the-art overview and future directions. Schizoph Bull 2012; 38: 724-33.

5 Fusar-Poli P, Nelson B, Valmaggia L, Yung AR, McGuire PK. Comorbid depressive and anxiety disorders in 509 individuals with an at-risk mental state: impact on psychopathology and transition to psychosis. Schizophr Bull 2014; 40: 120-31.

Rachel Upthegrove, Department of Psychiatry, School of Clinical \& Experimental Medicine, University of Birmingham, Birmingham, Bipolar Disorder Research Network and Early Intervention Service, Birmingham and Solihull Mental Health Foundation Trust, Birmingham, UK Email: R.Upthegrove@bham ac uk: Ian Jones, Nick Craddock, National Centre for Mental Health, MRC Centre for Neuropsychiatric Genetics and Genomics, Cardiff University, Cardiff and Bipolar Disorder Research Network, UK

doi: 10.1192/bjp.207.3.272

\section{Childhood non-affective psychoses: data analysis}

The epidemiology of psychosis in children is a story of even smaller numbers than for psychosis in grown-ups. But how much smaller? The short report of Tiffin \& Kitchen ${ }^{1}$ on incident cases of schizophrenia in children in 2010-2011 is a well-needed addition to a limited descriptive literature on psychosis in children. I have a couple of points about the analysis, which I feel might clarify the report.

The authors point out at the outset the heuristic that earlier onset cases of a disorder might have a 'greater loading of causal factors'. This does not seem to be correct - all cases of a given disorder represent the interrelation of a number of risk factors on a causal pathway - it is therefore unclear why earlier cases would represent the culmination of pathways with a greater number of causal factors. The authors may mean that earlier onset psychoses could represent individuals with stronger risk factors for the disorder. However, although this is usually correct for diseases in general, this point rests on the assumption that there is a single underlying outcome under study, which has long been contested. ${ }^{2}$ Assuming this heterogeneity is related in some way to the clinical heterogeneity of schizophrenia across people of different ages and with different risk factor patterns, this may explain the large number of false positives identified in Tiffin \& Kitchen's study.

Second, although the authors present confidence intervals (presumably based on Poisson standard errors), it is important to point out what the intervals are referring to - this study attempts to capture all cases within a surveillance approach, rather than to sample the target population. In this case, the confidence intervals refer to the underlying randomness of the disease process, rather than to the design of the study. ${ }^{3}$ Confidence intervals used in this way are not meaningless, but they may not be strictly necessary here - it seems that reporting the crude rate in this type of study is an almost equally informative approach for the purposes of this research. Furthermore, uncertainty about the true rate of the disorder due to random variability ('error') in the disease process likely pales in comparison with the systematic error introduced by biases in this design.

1 Tiffin PA, Kitchen CEW. Incidence and 12-month outcome of childhood nonaffective psychoses: British national surveillance study. Br J Psychiatry 2015; 206: $517-8$.

2 Tsuang MT, Lyons MJ, Faraone SV. Heterogeneity of schizophrenia. Conceptual models and analytic strategies. Br J Psychiatry 1990; 156 $17-26$.

3 Eayres D. Technical Briefing 3: Commonly Used Public Health Statistics and their Confidence Intervals. Association of Public Health Observatories, 2008.

Vishal Bhavsar, Epidemiologist, Institute of Psychiatry, Psychology and Neuroscience, King's College London, UK. Email: vishal.2.bhavsar@kcl.ac.uk

doi: 10.1192/bjp.207.3.272a

Authors' reply: To clarify, we did mean to state that earlier onset psychoses could represent individuals with stronger risk factors for the disorder. We agree that there is likely to be some heterogeneity in the outcomes, even if the loading of putative causal factors is similar for any set of given individuals. However, there is evidence that cases of childhood-onset psychosis spectrum disorders in general, when carefully defined, tend to be more severe and more homogeneous, with stronger family histories of schizophrenia spectrum disorders than adult-onset cases. ${ }^{1-3}$ Therefore, there may still be much to be learned about causality, even if the assumption of homogeneous clinical outcomes does not hold strictly true. Moreover, we believe the high rate of falsepositive reports is likely to be due, at least in most instances, to clinicians initially wrongly attributing perceptual disturbance in children to an underlying psychotic illness. In most cases, it is likely that voice experiences and other potentially psychotic phenomena may result from processes that may be conceptualised as more psychologically driven, such as dissociation. Such experiences are commonly reported in community samples of children and adolescents, who are likely to share few, if any, of the risk factors associated with the development of early-onset schizophrenia spectrum disorders. ${ }^{4}$ 